This document is the unedited Author's version of a Submitted Work that was subsequently accepted for publication in Chemistry of Materials, copyright (c) American Chemical Society after peer review. To access the final edited and published work see:

https://dx.doi. org/10.1021/acs.chemmater.7b03417. 


\title{
Unveiling a new high-temperature ordered magnetic phase in $\varepsilon-\mathrm{Fe}_{2} \mathrm{O}_{3}$
}

\author{
José Luis García-Muñoz ${ }^{1}$, Arnau Romaguera ${ }^{1}$, Francois Fauth ${ }^{2}$, Josep Nogués ${ }^{3,4}$ and \\ Martí Gich ${ }^{1}$
}

\footnotetext{
${ }^{1}$ Institut de Ciència de Materials de Barcelona, ICMAB-CSIC, Campus universitari de Bellaterra, E-08193 Bellaterra, Spain

2 ALBA Synchrotron Light Facility, 08290 Cerdanyola del Vallès, Barcelona, Spain

3 Catalan Institute of Nanoscience and Nanotechnology (ICN2), CSIC and The Barcelona Institute of Science and Technology, Campus UAB, Bellaterra, 08193 Barcelona, Spain.

${ }^{4}$ ICREA, Pg. Lluís Companys 23, 08010 Barcelona, Spain
}

PACS numbers: 75.25.-j; 75.85.+t; 75.50.Vv ; 75.47.Lx ; 75.50.Tt ; 75.50.Gg ; 77.55.Nv 


\begin{abstract}
Iron oxides are among the most abundant materials on earth and yet there are some of their basic properties which are still not well established. Here, we present temperature-dependent magnetic, X-ray and neutron diffraction measurements refuting the current belief that the magnetic ordering temperature of $\varepsilon-\mathrm{Fe}_{2} \mathrm{O}_{3}$ is $\sim 500 \mathrm{~K}$, i.e., well below that of other iron oxides such as hematite, magnetite or maghemite. Upon heating from room temperature, the saturation magnetization of $\varepsilon-\mathrm{Fe}_{2} \mathrm{O}_{3}$ nanoparticles undergoes a monotonic decrease while the coercivity and remanence sharply drop, virtually vanishing around $\sim 500 \mathrm{~K}$. However, above that temperature the hysteresis loops present a non-linear response with finite coercivity, evidencing signs of ferrimagnetic order up to temperatures as high as $850 \mathrm{~K}\left(\mathrm{~T}_{\mathrm{N} 1}\right)$. The neutron diffraction study confirms the presence of ferrimagnetic order well above $500 \mathrm{~K}$ with Pna'2 ${ }_{1}^{\prime}$ magnetic symmetry, but only involving two of the four $\mathrm{Fe}^{3+}$ sublattices which are ordered below $\mathrm{T}_{\mathrm{N} 2} \approx 480 \mathrm{~K}$, and with a reduced net ferromagnetic component, that vanishes at above $850 \mathrm{~K}$. The results unambiguously show the presence of a high-temperature magnetic phase in $\varepsilon-\mathrm{Fe}_{2} \mathrm{O}_{3}$ with a critical temperature of $\mathrm{T}_{\mathrm{N} 1} \sim 850 \mathrm{~K}$. Importantly, this temperature is similar to the Curie point in other iron oxides, indicating comparable magnetic coupling strengths. The presence of diverse magnetic phases is further supported by the nonmonotonic evolution of the thermal expansion. The existence of a high-temperature ferrimagnetic phase in $\varepsilon-\mathrm{Fe}_{2} \mathrm{O}_{3}$ may open the door to further expand the working range of this multifunctional iron oxide.
\end{abstract}




\section{INTRODUCTION}

Iron oxides constitute an exceptional family of materials that have been studied for many decades, because they present fundamental interest and proved and promising applicability for new technologies based on biochemical, magnetic, catalytic or multiferroic properties. ${ }^{1-4}$ Apart from the amorphous $\mathrm{Fe}_{2} \mathrm{O}_{3}$, Iron (III) oxide shows four known polymorph crystal structures: $\alpha-\mathrm{Fe}_{2} \mathrm{O}_{3}$ (hematite), $\beta-\mathrm{Fe}_{2} \mathrm{O}_{3}$ and $\gamma-\mathrm{Fe}_{2} \mathrm{O}_{3}$ (maghemite) and $\varepsilon-\mathrm{Fe}_{2} \mathrm{O}_{3} .{ }^{5}$ While hematite is the most common phase, $\varepsilon-\mathrm{Fe}_{2} \mathrm{O}_{3}$ is the most elusive and one of the less studied polymorphs. This is because its formation requires the mutually exclusive conditions of high temperatures and small sizes. ${ }^{6-8}$ The use of sol-gel methods with $\mathrm{Si}$ alcoxides and $\mathrm{Fe}$ salts made it possible to confine $\mathrm{Fe}_{2} \mathrm{O}_{3}$ nanoparticles in a silica matrix to prevent their growth while annealed above $1000^{\circ} \mathrm{C}$, allowing, for the first time, to obtain virtually pure $\varepsilon-\mathrm{Fe}_{2} \mathrm{O}_{3}$, opening the door to the study of this rare polymorph. ${ }^{9-11}$ The structural characterization evidenced a complex non-centrosymmetric Pna2 ${ }_{1}$ structure isomorphous to the multiferroic $\mathrm{GaFeO}_{3}$, with four $\mathrm{Fe}$ sites in the asymmetrical unit: three in octahedral and one in tetrahedral environments. ${ }^{8}$ The magnetic studies revealed an unexpected huge room temperature coercivity: $20 \mathrm{kOe}$, making it the transition metal oxide with the highest coercivity and an appealing material for the next-generation high-density magnetic recording media, highfrequency electromagnetic wave absorbers or a building block for exchange coupled permanent magnets. ${ }^{12-15}$ Moreover, other important functionalities of $\varepsilon-\mathrm{Fe}_{2} \mathrm{O}_{3}$ are related with its magnetoelectric ${ }^{16}$ and multiferroic properties. ${ }^{4}$ These properties, quite unique among single metal oxides, stem from the structural features specific to this polymorph, in spite of being considered a structural intermediate between $\alpha-\mathrm{Fe}_{2} \mathrm{O}_{3}$ and $\gamma-\mathrm{Fe}_{2} \mathrm{O}_{3}$. Its structure produces a rich temperature-dependent diagram of magnetic phases. Between $\mathrm{T}_{\mathrm{C}}=500 \mathrm{~K}$ and $\mathrm{T}_{\mathrm{N} 2}=150$ $\mathrm{K}, \varepsilon-\mathrm{Fe}_{2} \mathrm{O}_{3}$ exhibits a collinear ferrimagnetic structure due to the antiferromagnetic (AF) coupling (parallel to the $a$-axis) between dissimilar magnetic sublattices. This oxide then presents a broad low-temperature incommensurate magnetic transition starting at $150 \mathrm{~K}$ $(\mathrm{ICOM} 1)^{17-18}$, characterized by a large reduction of the coercivity, the remanent and the saturation magnetization ${ }^{17}$. Under further cooling, below $\mathrm{T}_{\mathrm{N} 3} \approx 85 \mathrm{~K}$, a second incommensurate magnetic order (ICOM2) takes place. ${ }^{13}$ Noticeable anomalies in the dielectric permittivity were reported in conjunction with these two successive transitions ${ }^{16}$. In addition, significant magnetostriction effects were observed in the interval 85-150 K where magnetic changes take place and spin-lattice coupling brings about atomic shifts within the 
orthorhombic cell and a non-monotonous evolution of the $\mathrm{Fe}-\mathrm{O}$ bonds ${ }^{19}$. A nonzero orbital magnetic moment $\left(\mathrm{m}_{\mathrm{L}} \sim 0.15 \mu_{\mathrm{B}} / \mathrm{Fe}\right.$ at $\mathrm{RT}$ under $\left.40 \mathrm{kOe}\right)$ was detected by $\mathrm{x}$-ray magnetic circular dichroism (XMCD) in the hard collinear ferrimagnetic phase $(150 \mathrm{~K}<\mathrm{T}<490 \mathrm{~K})$, which is suppressed by the magnetostructural changes concurrent with the magnetic transitions within the $85-150 \mathrm{~K}$ interval. ${ }^{15}$ On cooling, the coercive field drops in that interval from $20 \mathrm{kOe}$ to $0.8 \mathrm{kOe}$, and then increases again under further cooling in the lowtemperature magnetic phase (ICOM2), below $85 \mathrm{~K} .{ }^{14}$ Likewise, a reentrant behavior of the orbital moment was also monitored by XMCD in the low temperature magnetic phase. ${ }^{14}$ The concurrent magnetostructural changes in the coordination polyhedra around $\mathrm{Fe}^{3+}$ sites and the reentrant evolution of the orbital moment should be seen as the signature of a relevant spinorbit coupling in this magnetoelectric compound. Finally, the RT coexistence of ferrimagnetism and ferroelectricity, albeit with a small magnetocapacitance response, in thin epitaxial films of $\varepsilon-\mathrm{Fe}_{2} \mathrm{O}_{3}$ further highlights the relevance of magnetoelastic couplings in this system, for which possible multiferroic properties in bulk form are still a subject of debate.

Importantly, although the Curie temperature of $\varepsilon-\mathrm{Fe}_{2} \mathrm{O}_{3}$ has been postulated as $\mathrm{T}_{\mathrm{C}} \sim 500 \mathrm{~K},{ }^{3}$, 11, 20-23 no systematic studies of the magnetic properties above this temperature can be found in the literature.

Using diffraction and magnetometry techniques, we have investigated the magnetic and magnetostructural properties of $\varepsilon-\mathrm{Fe}_{2} \mathrm{O}_{3}$ nanoparticles at high temperatures $(\mathrm{T}>500 \mathrm{~K})$. The results evidence that contrary to the established scenario, $\varepsilon-\mathrm{Fe}_{2} \mathrm{O}_{3}$ remains ferrimagnetic up to at least $\mathrm{T}=850 \mathrm{~K}$, although with a reduced magnetization and a moderate coercivity.

\section{EXPERIMENTAL DETAILS}

In order to have a good signal to noise ratio in neutron diffraction studies it is desirable to perform measurements using more than $1.5 \mathrm{~g}$ of $\varepsilon-\mathrm{Fe}_{2} \mathrm{O}_{3}$ (ideally obtained in a single synthesis). Thus, we prepared a silica gel containing 28 wt. $\% \mathrm{Fe}_{2} \mathrm{O}_{3}$ from an hidroethanolic sol of tetraethyl orthosilicate (TEOS) of molar composition TEOS:Ethanol:water=1:5:6 containing dissolved iron nitrate nonahydrate. First, $5.4 \mathrm{ml}$ of milliQ water and $26 \mathrm{ml}$ of absolut ethanol (Panreac) were added to a $100 \mathrm{ml}$ beaker and stirred for $5 \mathrm{~min}$. Then, the $10.60 \mathrm{~g}$ of iron nitrate (Aldrich) were dissolved and the solution which attained a $\mathrm{pH} \sim 0.35$. Finally $20 \mathrm{ml}$ of TEOS (Aldrich) were added dropwise to the solution under stirring ( 200 $\mathrm{rpm})$. The stirring, with the beaker covered, was maintained for 20 minutes after adding the TEOS. The sol was then distributed in $6 \mathrm{~cm}$ diameter petri dishes, attaining a level of 2-4 
$\mathrm{mm}$, which were placed in a plastic box, closed with its cover but not hermetically sealed, and placed in chemical hood at $23{ }^{\circ} \mathrm{C}$. Gelation took place in about two weeks. The gels were removed from the petri dishes, allowed to dry and subsequently grinded in a ceramic mortar to be further dried at $60^{\circ} \mathrm{C}$ in a stove. Then the xerogels were placed in an alumina boat and treated in a tubular furnace in air atmosphere at $200^{\circ} \mathrm{C} / \mathrm{h}$ to $450^{\circ} \mathrm{C}$ and then to $1100^{\circ} \mathrm{C}$ at 80 ${ }^{\circ} \mathrm{C} / \mathrm{h}$, and were held for $3 \mathrm{~h}$ at this temperature before being cooled to room temperature at $350{ }^{\circ} \mathrm{C} / \mathrm{h}$. About $7.5 \mathrm{~g}$ of $\mathrm{SiO}_{2} / \varepsilon-\mathrm{Fe}_{2} \mathrm{O}_{3}$ composite were obtained. The resulting material consisted of single crystalline $\varepsilon-\mathrm{Fe}_{2} \mathrm{O}_{3}$ nanoparticles embedded in a silica matrix with an average diameter of around $20 \mathrm{~nm}$ as observed by transmission electron microscopy. The silica was etched in hot $\left(80^{\circ} \mathrm{C}\right)$ concentrated $\mathrm{NaOH}$ aqueous solution $(12 \mathrm{M})$. For this purpose a round bottomed flask was filled with $180 \mathrm{ml}$ of distilled water in which $76 \mathrm{~g} \mathrm{NaOH}$ were dissolved, then about $5 \mathrm{~g}$ of $\mathrm{SiO}_{2} / \varepsilon-\mathrm{Fe}_{2} \mathrm{O}_{3}$ composite were added to it and stirred overnight in a hot plate set at $80^{\circ} \mathrm{C}$ using a water refrigerated condenser to avoid evaporation of water and a silicone oil bath to maintain an homogeneous temperature around the flask. Then the suspension was centrifuged at $6000 \mathrm{rpm}$ for $2 \mathrm{~min}$ and the supernatant was discarded. The collected solid was re-dispersed in water and the centrifugation was repeated twice. Finally the tubes used for centrifugation were placed in a stove at $60^{\circ} \mathrm{C}$ and about $1.5 \mathrm{~g}$ of $\varepsilon-\mathrm{Fe}_{2} \mathrm{O}_{3}$ nanoparticles were collected after drying.

The quality of the samples was assessed by transmission electron microscopy, magnetic measurements and $\mathrm{x}$-ray diffraction, as reported earlier ${ }^{6,}{ }^{17}$. Synchrotron $\mathrm{X}$-ray powder diffraction patterns (SXRPD) were collected at the BL04-MSPD beamline ${ }^{24}$ of the ALBA Synchrotron Light Facility (Barcelona, Spain) using $\lambda=0.41284(6) \AA$. Patterns were recorded every $90 \mathrm{~s}$ by the MYTHEN position sensitive detector while warming the sample from 300 up to $923 \mathrm{~K}$ at a rate of $3 \mathrm{~K} / \mathrm{min}$. The working temperature was set using a CYBERSTAR hot air blower. Neutron powder diffraction (NPD) patterns were collected using the highintensity, high-resolution D2B diffractometer of the Institute Laue-Langevin (ILL, Grenoble), between room temperature (RT) and $850 \mathrm{~K}(\lambda=1.594 \AA$ ). Structural and magnetic Rietveld refinements were carried out using the Fullprof program ${ }^{25}$. Crystallographic tools from the Bilbao Crystallographic server ${ }^{26-28}$ and ISOTROPY Software Suite ${ }^{29}$ were also used.

The magnetic characterization, using dc and ac magnetic fields, was performed with the asprepared $\varepsilon-\mathrm{Fe}_{2} \mathrm{O}_{3}$ nanoparticles embedded in $\mathrm{SiO}_{2}$, using a Superconducting Quantum Interferometer Device (SQUID) and a Vibrating Sample Magnetometer (VSM) in a Physical Properties Measuring System (PPMS) both from Quantum Design. For the VSM 
measurements the $\varepsilon-\mathrm{Fe}_{2} \mathrm{O}_{3} / \mathrm{SiO}_{2}$ material was mixed with a high-temperature alumina cement. The use of $\varepsilon-\mathrm{Fe}_{2} \mathrm{O}_{3} / \mathrm{SiO}_{2}$ composite instead of $\varepsilon-\mathrm{Fe}_{2} \mathrm{O}_{3}$ was necessary to avoid the transformation of $\varepsilon-\mathrm{Fe}_{2} \mathrm{O}_{3}$ to magnetite due to high temperatures and the vacuum environment during the measurements. The ac-susceptibility measurements were performed at $30 \mathrm{~Hz}$ and $1 \mathrm{kHz}$ with a magnetic field amplitude of $4 \mathrm{Oe}$ from $300 \mathrm{~K}$ to $750 \mathrm{~K}$. The temperature dependence of the magnetization, M, was studied after zero field cooled (ZFC) and FC conditions under a dc field of $1 \mathrm{kOe}$ from 300 to $900 \mathrm{~K}$. Hysteresis loops were obtained between $900 \mathrm{~K}$ and $300 \mathrm{~K}$ with a maximum applied magnetic field of $70 \mathrm{kOe}$.

\section{RESULTS AND DISCUSSION}

First it is worth emphasizing that the orthorhombic Pna2 ${ }_{1}$ structure was stable up to the highest temperature reached $(923 \mathrm{~K})$. Namely, no transition to other more stable iron oxides (e.g., $\alpha-\mathrm{Fe}_{2} \mathrm{O}_{3}$ or $\gamma-\mathrm{Fe}_{2} \mathrm{O}_{3}$ ) has been observed up to that temperature. The structural parameters of $\varepsilon-\mathrm{Fe}_{2} \mathrm{O}_{3}$ and agreement factors from the refinement of SXRPD patterns obtained at $305 \mathrm{~K}$ and $510 \mathrm{~K}$ can be seen in Table I. Figure 1(a) displays a projection of the $\varepsilon$ $\mathrm{Fe}_{2} \mathrm{O}_{3}$ non-centrosymmetric Pna2 ${ }_{1}$ structure, composed of four different $\mathrm{Fe}$ sites in the asymmetrical unit cell, three octahedral and one tetrahedral environments. The coordination octahedra $\mathrm{Fe} 1 \mathrm{O}_{6}$ and $\mathrm{Fe} 2 \mathrm{O}_{6}$ are largely distorted, $\mathrm{Fe} 3 \mathrm{O}_{6}$ is a regular octahedron and $\mathrm{Fe} 4 \mathrm{O}_{4}$ is the tetrahedron. For clarity we will use the Fe1d, Fe2d, Fe3r, Fe4t atomic labels where "d", " $\mathrm{r}$ " and " $\mathrm{t}$ " refer to "distorted", "regular" and "tetrahedral" polyhedral coordination, respectively.

The presence of very minority impurities was carefully explored by a detailed examination of high intensity synchrotron patterns. We detected a 2.3(9)\% in weight of $\alpha-\mathrm{Fe}_{2} \mathrm{O}_{3}$ (hematite) as impurity phase in our nanograin ceramic samples of $\varepsilon-\mathrm{Fe}_{2} \mathrm{O}_{3}$ (corresponding to the bottom row of bars in the refined synchrotron pattern shown in Fig. 1(b)). This corresponds to a 0.7 wt. \% in the $\mathrm{SiO}_{2} / \mathrm{Fe}_{2} \mathrm{O}_{3}$ composite used for magnetic measurements. 

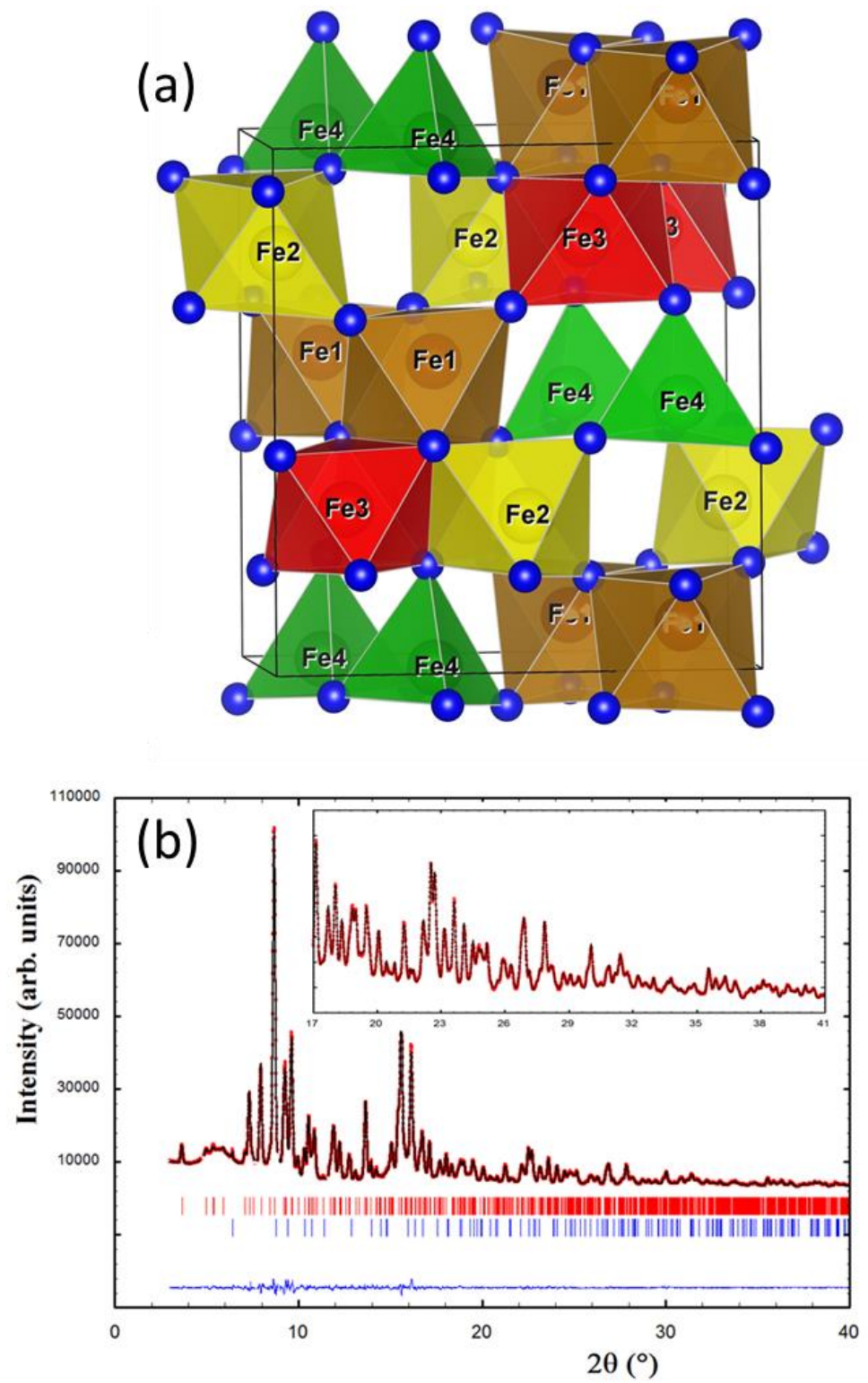

Figure 1. (a) Crystallographic projection of $\varepsilon-\mathrm{Fe}_{2} \mathrm{O}_{3}$. The four crystallographically independent $\mathrm{Fe}$ sites are shown with different colors in the structure (Fe1, brown; $\mathrm{Fe} 2$ yellow; $\mathrm{Fe} 3$ green; $\mathrm{Fe} 4$ red). $\mathrm{O}$ atoms are represented in dark blue. (b) Rietveld refinement (black solid line) of the synchrotron x-ray pattern of $\varepsilon-\mathrm{Fe}_{2} \mathrm{O}_{3}$ collected at $300 \mathrm{~K}$ (red circles: experimental points; bottom blue line: difference). The top row of bars (in red) corresponds to $\varepsilon-\mathrm{Fe}_{2} \mathrm{O}_{3}$, while the bottom row (in blue) is for hematite $\left(\alpha-\mathrm{Fe}_{2} \mathrm{O}_{3}, 2 \%\right.$ weight). The inset shows an enlarged view of the high-angle region.

\section{III-a Magnetometry}

The temperature dependence of the ZFC and $\mathrm{FC}$ magnetization of the $\varepsilon-\mathrm{Fe}_{2} \mathrm{O}_{3}$ particles embedded in $\mathrm{SiO}_{2}$ is plotted in Fig. 2(a). Two ferrimagnetic regimes with very different magnetic behavior can be clearly distinguished below and above $500 \mathrm{~K}$ (denoted FM2 and FM1 respectively). Remarkably, contrary to the scenario assumed in previous reports, the 
ferrimagnetic FM2 phase does not become paramagnetic above $500 \mathrm{~K}$. The $M(T)$ evolution in Fig. 2 reveals a second phase transition, evidencing the existence of a new ferrimagnetic state (FM1) between $500 \mathrm{~K}$ and $\sim 850 \mathrm{~K}$. The transition temperature between FM1 and FM2 can be established at $T_{N 2}=480 \mathrm{~K}$ by the lambda-shaped peak in the ac susceptibility vs temperature curve presented in the inset of Fig. 2(a). From the hysteresis loops at $T>500 \mathrm{~K}$ it can be clearly seen that FM1 presents a ferromagnetic behavior with finite net magnetic moment and coercivity, $H_{C}$.
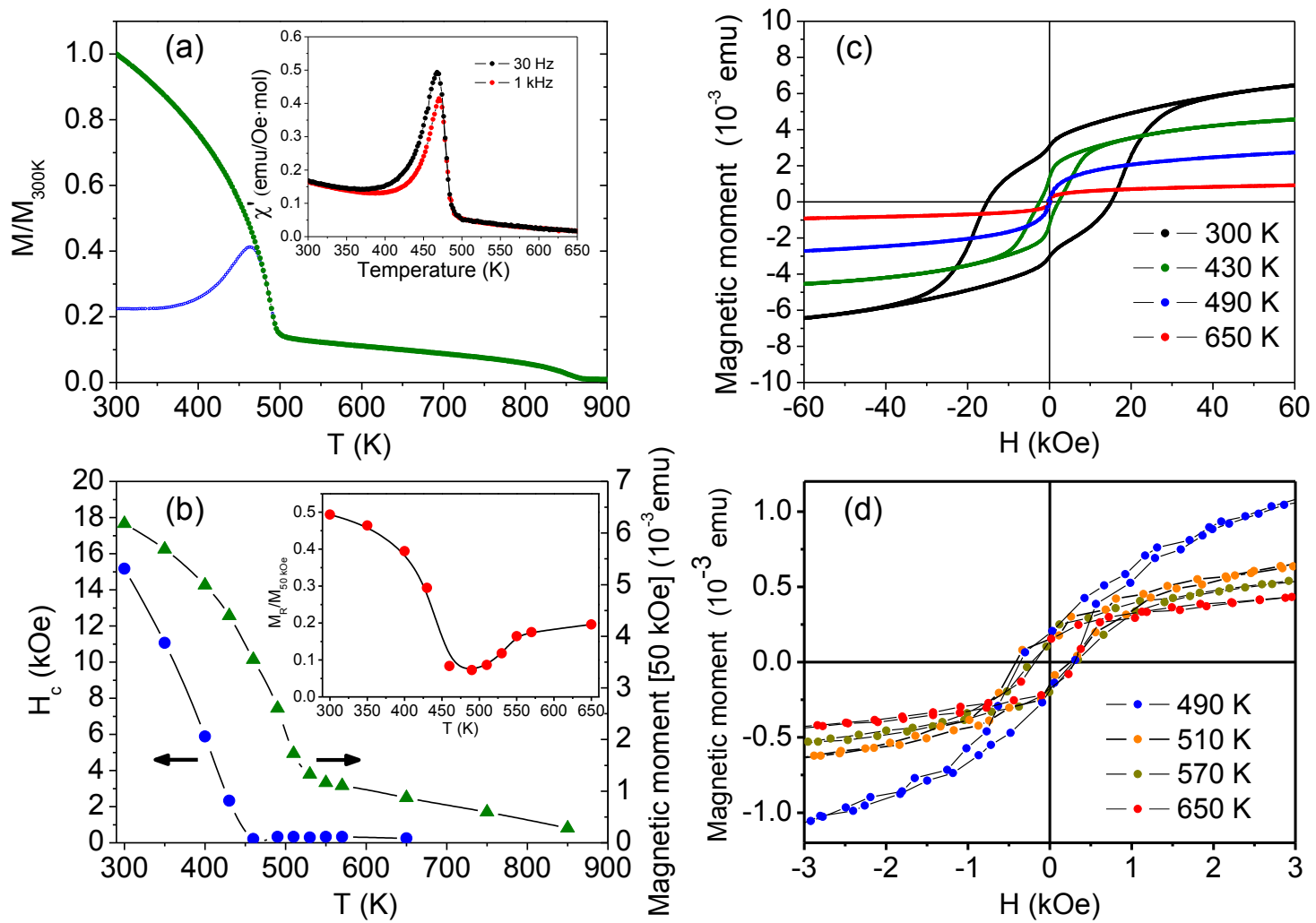

Figure 2. (Color online) (a) FC (green) and ZFC (blue) dc magnetization curves $(1 \mathrm{kOe})$ of the $\varepsilon$ $\mathrm{Fe}_{2} \mathrm{O}_{3}$ nanoparticles embedded in $\mathrm{SiO}_{2}$ between 300 and $900 \mathrm{~K}$. Inset: real part of the ac-susceptibility $(\mathrm{h}=4 \mathrm{Oe})$. (b) Temperature evolution of the coercive field $\left(H_{C}\right.$; left axis) and net magnetic moment at $50 \mathrm{kOe}\left(M[50 \mathrm{kOe}]\right.$; right axis). Inset: dependence of the $M_{r} / M[50 \mathrm{kOe}]$ ratio. Hysteresis loops characteristic of (c) the FM2 phase and (d) the FM1 phase (note the different $\mathrm{x}$ - and y-scales in both figures, and the loops at $490 \mathrm{~K}$ and $650 \mathrm{~K}$ included in both panels for the sake of comparison).

However, as can be seen in Fig. 2(b) there is a drastic collapse of the large $H_{C}$ (characteristic of FM2) and $M[50 \mathrm{kOe}]$ at $T_{N 2}=480 \mathrm{~K}$ (see Figs. 2(b)(c)). Moreover, upon heating, $M[50$ kOe] undergoes a Brillouin-type monotonic decrease up to $550 \mathrm{~K}$, although the coercivity (and remanence, $M_{r}$ ) sharply drop well before this temperature, having practically vanished around $\sim 480 \mathrm{~K}$. Nevertheless, although $H_{C}$ shrinks by more than a factor 10 from $H_{C} \sim 16$ 
$\mathrm{kOe}$ at RT, it remains moderate $(\sim 400 \mathrm{Oe})$ even at $T \gg>500 \mathrm{~K}\left(T_{N 2}\right)$ (see Fig. $2(\mathrm{~d})$ ). Importantly, from the evolution of the magnetic properties it can be established that the critical temperature of the FM1 phase is about $T_{N 1} \sim 850 \mathrm{~K}$. Hence, although there is a drastic breakdown of the hard ferrimagnetic state at $T_{N 2}=480 \mathrm{~K}$, it does not give rise to a paramagnetic phase, but it is transformed into a new softer ferrimagnetic state.
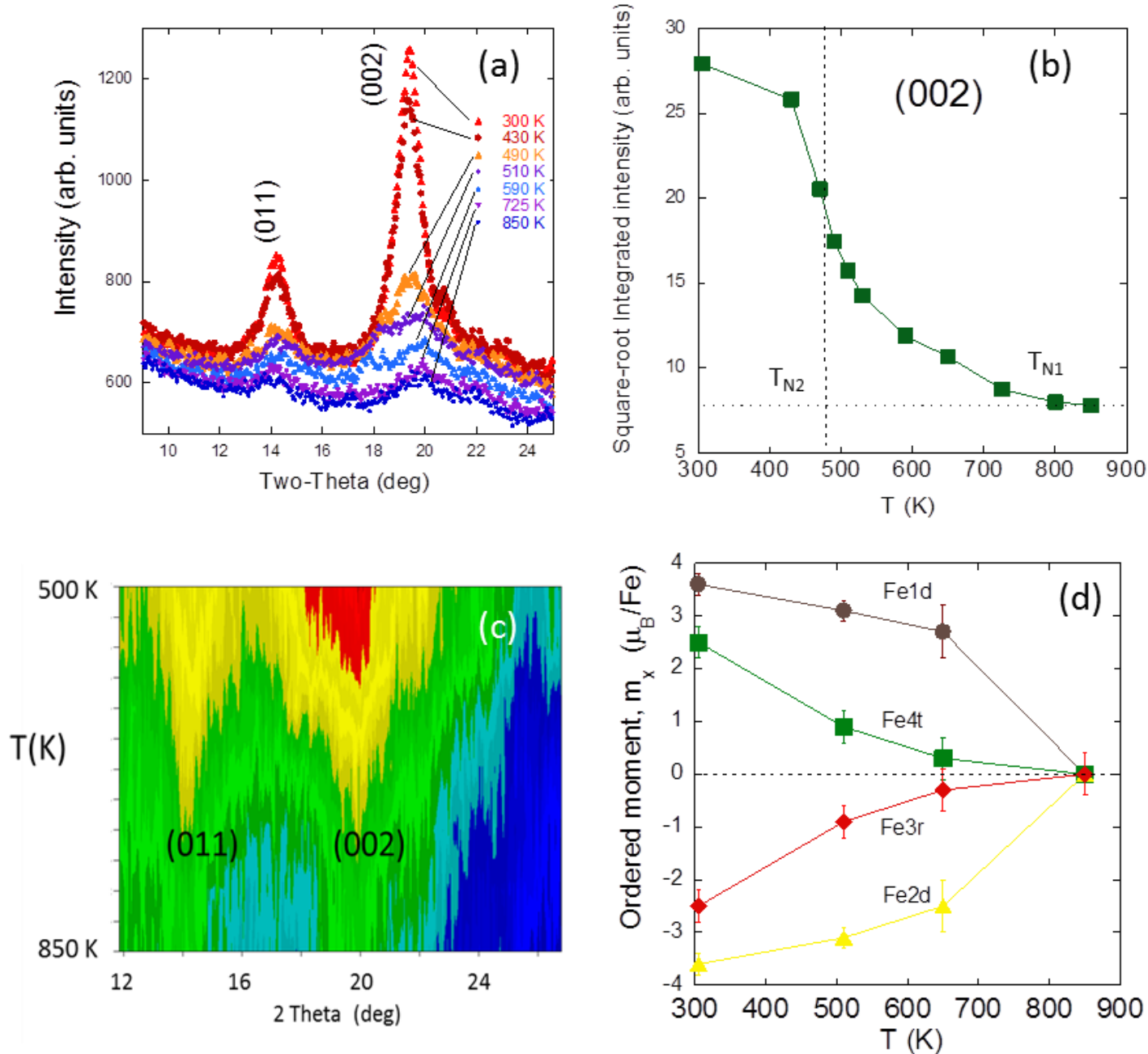

Figure 3. (a) Neutron patterns at selected temperatures in the range comprising the main magnetic peaks (011) and (002). (b) Square-root of the neutron integrated intensity of the strongest magnetic reflection (002). (c) Evolution of the neutron diffraction intensities above $\mathrm{T}_{\mathrm{N} 2}$ for the characteristic magnetic reflections. (d) Evolution of the ordered magnetic moments refined at Fe1d (brown), Fe2d (yellow), Fe3r (red) and Fe4t (green) sites in the structure. 


\section{III-b Neutron diffraction study of the successive ferrimagnetic phases}

To better understand the nature of the very high-temperature ferrimagnetic phase a systematic NPD study was performed.

As can be seen in Fig. 3(a), the intensity of the magnetic peaks (011) and (002) progressively decreases as the temperature is increased from RT to $850 \mathrm{~K}$. In agreement with the magnetization results, the intensity of the magnetic peaks remains finite even far above $T_{N 2}$. However, the temperature dependence of the integrated intensity of the (002) peak (proportional to the ordered moment), shown in Fig. 3(b), strongly differs from a Brillouintype evolution and exhibits the shape of a long magnetic tail that persists far beyond $\mathrm{T}_{\mathrm{N} 2}$.

The magnetic structures and atomic ordered moments were fully analyzed using the neutron diffraction patterns at the two selected temperatures $305 \mathrm{~K}$ and $510 \mathrm{~K}$. The former being representative of the ferrimagnetic phase FM2, and the second (above but close to $T_{N 2}=480$ $\mathrm{K})$ of the new ferrimagnetic phase FM1. Note that the magnetic reflections detected between $T_{N 2}(=480 \mathrm{~K})$ and $T_{N 1}(\sim 850 \mathrm{~K})$ do not indicate changes in the extinction conditions or the translational symmetry.

Possible magnetic or Shubnikov space groups compatible with the Pna2 ${ }_{1}$ symmetry and null magnetic propagation vector $\mathbf{k}=0$ were considered. It was found that the magnetic ordering adopts the same magnetic space group in the two ferrimagnetic phases, Pna' $2_{1}$ ' [\# 33.147, transformation to standard settings: (a, b, c; 0, 0, 0) $]^{25,27}$. The Rietveld refinement of neutron patterns at $305 \mathrm{~K}$ (FM2 magnetic phase) and $510 \mathrm{~K}$ (FM1 magnetic phase) are plotted in Figure 4 and the results are summarized in Table II. 

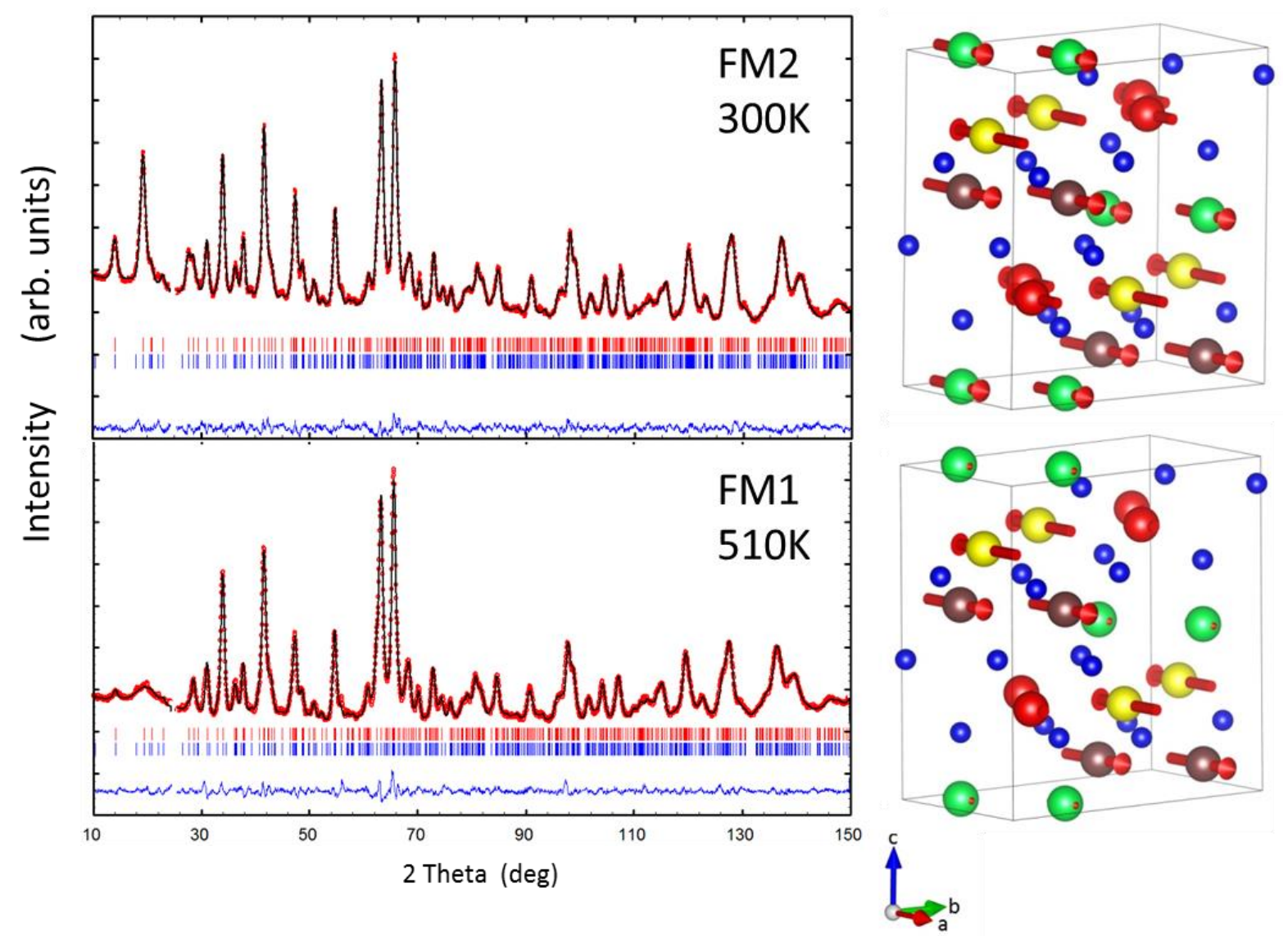

Figure 4. (Color online) Neutron Rietveld refinement (black line) of the NPD patterns for $\varepsilon-\mathrm{Fe}_{2} \mathrm{O}_{3}$ obtained at $305 \mathrm{~K}\left(T<T_{N 2}, \mathrm{FM} 2\right.$ ordering) and $510 \mathrm{~K}\left(T_{N 2}<T<T_{N 1}\right.$, FM1 ordering) (D2B, red circles: experimental points; bottom blue line: difference). Upper row of reflections correspond to the Pna2 1 structure, the lower one to the magnetic ordered phase (Pna' $\left.21^{\prime}\right)$. Goodness factors at $305 \mathrm{~K}(510 \mathrm{~K})$ : $\mathrm{R}_{\mathrm{B}}=2.2$ 2.48(1.8), $\mathrm{R}_{\mathrm{f}}=1.3$ 1.55(1.1), $\mathrm{R}_{\mathrm{Mag}}=2.2$ 2.92(1.2), $\chi^{2}=1.27$ 0.31 (3.5). (Right) Schematic view of the FM1 (bottom) and FM2 (top) magnetic ordering at the same temperatures. Fe1d (brown), Fe2d (yellow), $\mathrm{Fe} 3 \mathrm{r}$ (red) and $\mathrm{Fe} 4 \mathrm{t}$ (green)

Interestingly, despite the abrupt drop in the displayed by magnetization at $T_{N 2}$, there are ordered magnetic moments with very large $\mathrm{m}_{\mathrm{x}}$ components that steadily persist in the interval $T_{N 2}(=480 \mathrm{~K})<T<T_{N 1}(=850 \mathrm{~K})(\mathrm{m}$, magnetic moment $)$. Nevertheless, they are found selectively in Fe1d and Fe2d octahedral sites. These two Fe positions are magnetically robust and persist antiferromagnetically coupled above $T_{N 2}$. Their antiparallel ordered moments as refined at intermediate temperatures have been plotted in Fig. 3(d). Remarkably, the main difference detected between FM2 and FM1 orders concerns the high degree of magnetic disorder at the $\mathrm{Fe} 3 \mathrm{rO} 6$ and $\mathrm{Fe} 4 \mathrm{tO} 4$ sites. Neutron data did not allow us discern ordered moments at $\mathrm{Fe} 3 \mathrm{r}$ and $\mathrm{Fe} 4 \mathrm{t}$ sites independently, and their moments were kept (above $T_{N 2}$ ) identical and antiparallel, forcing $m_{x}[\mathrm{Fe} 3 r]=-m_{x}[\mathrm{Fe} 4 t]$, in the neutron refinements. The 
refined $m_{x}$ moments at these positions are also displayed in the Fig. 3(d), which shows the evolution of the ordered moments $\left(\mathrm{m}_{\mathrm{x}}\right)$ refined for the four Fe sites. In the high temperature phase the difference between the moments in one antiparallel pair (e.g. Fe3r/Fe4t) is smaller than our experimental error. At RT $(305 \mathrm{~K})$ the resultant net uncompensated moment $\sim 0.2$ $\mu_{\mathrm{B}} / \mathrm{Fe}$ (i.e. $14 \mathrm{emu} / \mathrm{g}$ ) is in agreement with $\mathrm{M}_{\mathrm{s}}$ values (net ferromagnetic signal) previously reported ${ }^{10,17}$. Therefore, at the FM2/FM1 (hard/soft) ferrimagnetic phase boundary, there is a clear disruption of the magnetic order of the iron spins occupying the tetrahedral Fe4t and the undistorted octahedral $\mathrm{Fe} 3 \mathrm{r}$ sites. The small moment values refined at $510 \mathrm{~K}$ indicate that $\mathrm{Fe} 3 \mathrm{r}$ and Fe4t sublattices are practically disordered above $T_{N 2}$. Likewise, the onset of magnetic ordering near $850 \mathrm{~K}$ is essentially driven by the AFM coupling of Fe spins in the more distorted octahedral positions (Fe1d and Fe2d). Fig. 3(d) clearly illustrates the anomalous evolution of the ordered moments at $\mathrm{Fe} 3 \mathrm{r} / \mathrm{Fe} 4 \mathrm{t}$ sites. Most likely this evolution is the result of strong frustration effects between sublattices, which can be identified by the broad magnetic reflections at high temperatures.

Using a molecular-field model, the appearance of spontaneous magnetization in the FM2 phase of $\varepsilon-\mathrm{Fe}_{2} \mathrm{O}_{3}$ has been associated with the lower superexchange $Z_{i j} J_{i j}$ values of the tetrahedral site, compared to those of octahedral sites. ${ }^{22}$ As can be seen in Fig. $3 \mathrm{~d}$ the ordered moments at the Fe1d and Fe2d sites are larger than in the Fe4t tetragonal site in the whole range above room temperature, in concordance with the theoretical predictions. On another hand, our neutron diffraction results establish that the abrupt enhancement of the magnetization below $T_{N 2}$ takes place concurrently with the long-range ordering of $\mathrm{Fe} 4 \mathrm{t}$ magnetic atoms occupying the tetrahedral site.

Interestingly, the new ferrimagnetic-paramagnetic boundary is shifted towards the upper characteristic ordering temperatures of ferrimagnetic iron oxides, $950 \mathrm{~K}$ for $\alpha-\mathrm{Fe}_{2} \mathrm{O}_{3}, 940 \mathrm{~K}$ for $\gamma-\mathrm{Fe}_{2} \mathrm{O}_{3}$ and $853 \mathrm{~K}$ for $\mathrm{Fe}_{3} \mathrm{O}_{4}$, indicating that $\varepsilon-\mathrm{Fe}_{2} \mathrm{O}_{3}$ presents comparable magnetic coupling strengths. Notably, the NPD analysis unambiguously demonstrates that the ordered magnetic components are associated to $\varepsilon-\mathrm{Fe}_{2} \mathrm{O}_{3}$, ruling out the possibility that the magnetic response above $480 \mathrm{~K}$ might be due to impurities of the ferrimagnetic oxides mentioned above, which present a completely different set of magnetic reflections (see Fig. S1 of the Supplementary Material). Additionally, the fact that some of the magnetic peaks, although very broad, remain finite at $850 \mathrm{~K}$ may indicate the possible existence of a frustrated (with no long-range order) ferrimagnetic phase even above $T_{N 1}$, similar to other oxide systems. ${ }^{30}$ 


\section{III-c Magneto-structural coupling at the ferrimagnetic transitions studied using synchrotron $x$-ray diffraction}

Magnetic ordering at high temperatures in $\varepsilon-\mathrm{Fe}_{2} \mathrm{O}_{3}$ concurs with significant magnetostructural and thermal expansion anomalies, as shown by the evolution of the orthorhombic cell obtained from SXRPD (Figure 5). The cell volume reveals an abrupt contraction $(\approx-0.1 \%)$ at about $500 \mathrm{~K}$ upon cooling from high temperature (Fig. 5(a)) with the appearance of the hard FM2 phase. The anomaly is also visible in the evolution of all three $a(T), b(T)$ and $c(T)$ cell parameters (Fig. 5(b)): $a$ and $b$ abruptly contract and $c$ expands when Fe3r and Fe4t spins become long-range ordered $\left(T_{N 2}\right)$.
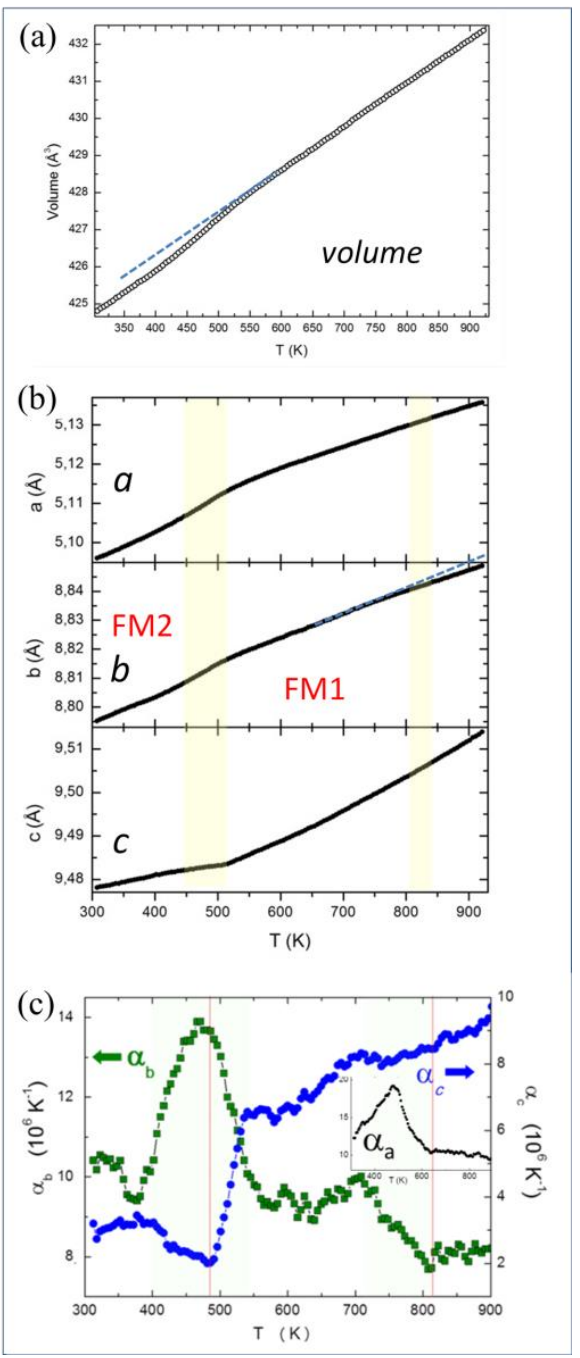

Figure 5. (a) Evolution of the unit-cell volume above room temperature obtained from SXRPD data. (b) Temperature dependence of the orthorhombic cell parameters in the 300-925 K range showing magnetostructural anomalies associated to the emergence of the FM1 and FM2 ferrimagnetic orders. (c) The linear thermal expansion coefficient $\alpha_{i}\left(\mathrm{i}=\mathrm{b}\right.$ and c) derived from SXRPD data and $a_{i}(\mathrm{~T})$ as $\alpha_{i}=1 / l_{i 0} \mathrm{x} \mathrm{d} l_{i} / \mathrm{dT}$. The temperature dependence of the coefficients $\alpha_{b}(\mathrm{~T})$ and $\alpha_{c}(\mathrm{~T})$ are shown on the left- and right-axis respectively. Inset: $\alpha_{a}(\mathrm{~T})$. 
In the Fig. 5(c) we plot the linear thermal expansion coefficients $\alpha$, deduced from $\alpha_{i}(\mathrm{~T})$ as $\alpha_{i}$ $=1 / l_{\mathrm{i} 0} \times \mathrm{d} l_{i} / \mathrm{dT}\left(l_{\mathrm{i}}: a, b, c\right)$, where $l_{\mathrm{i} 0}$ is the value of the $l_{\mathrm{i}}$ parameter (size of the unit cell along $\mathrm{i}: a, b, c)$ at $305 \mathrm{~K}$. Interestingly, there are anomalous contributions to the lattice evolution which are concurrent with the magnetic transitions. On the one hand, the most prominent exchange-striction effects come up accompanying the hard/soft ferrimagnetic phase boundary (Fig. 2). Similarly, the evolution of $\alpha_{b}(\mathrm{~T})$ in Fig. 5(c) also shows a significant anomaly in the range 700-825 K which could be caused by the appearance of magnetic order at Fe1d and Fe2d sites in the FM1 phase, which is in concordance with the small deviation observed in $b(\mathrm{~T})$ near $\sim 800 \mathrm{~K}$ (Fig. 5(b)). Note that the exchange-striction effect at $\mathrm{T}_{\mathrm{N} 1}$ provides further evidence that the magnetic properties between $480 \mathrm{~K}$ and $850 \mathrm{~K}$ do not originate from impurities, but from the $\mathrm{FM} 1$ phase of $\varepsilon-\mathrm{Fe}_{2} \mathrm{O}_{3}$.

In this way, although both ferrimagnetic phases exhibit the same magnetic space group $\left(P_{n}^{\prime} 2_{1}{ }^{\prime}\right.$ (n. 33.147)) and moment orientation (parallel to $a$ ), the observed magnetostructural response is very different in both transitions. The origin of these differences should correlate with the dissimilar character of each of the phases (i.e., fully ordered FM2 vs partially frustrated FM1).

\section{CONCLUDING REMARKS}

In the preceding sections we have investigated the puzzling magneto-structural properties of polycrystalline $\varepsilon-\mathrm{Fe}_{2} \mathrm{O}_{3}$ above room temperature. Contrary to the scenario proposed in the literature, magnetic order in this magnetoelectric Iron (III) polymorph does not disappear at the hard-ferrimagnetic transition near $480 \mathrm{~K}$. We have shown that a second ferrimagnetic phase (FM1) persists up to much higher temperatures (near $850 \mathrm{~K}$ ). The hard ferrimagnetic FM2 phase (holding giant coercivity presumably stimulated by a nonzero orbital angular moment) is transformed to a different ferrimagnet state, FM1, with a much smaller ferromagnetic component and coercivity. Both magnetic orders adopt the magnetic space group $P n a^{\prime}{ }_{1}{ }^{\prime}$ (n. 33.147), where the main difference between them is the disruption of the magnetic order associated to the Fe3r and Fe4t magnetic atoms. Above $480 \mathrm{~K}$, the iron spins at the sublattices that occupy the regular octahedra $\mathrm{Fe} 3 \mathrm{O}_{6}$ and $\mathrm{Fe} 4 \mathrm{O}_{4}$ tetrahedra can hardly keep their antiparallel magnetizations and become rapidly disordered. Consequently, the spontaneous FM magnetization that was sustained by the 
different magnetic moments in these antiparallel sublattices in FM2 quickly vanishes in FM1. Thus, the small FM component persisting up to $T_{N 1} \approx 850 \mathrm{~K}$ results from a slightly dissimilar magnetization at the antiparallel Fe1d and Fe2d sublattices. Hence, we have demonstrated that the ferrimagnetic-paramagnetic phase boundary in $\varepsilon-\mathrm{Fe}_{2} \mathrm{O}_{3}$ is $T_{N 1} \sim 850$ $\mathrm{K}$. This temperature is similar to the Curie point in other iron oxides, confirming comparable magnetic coupling strengths. This may open the door to further expand the working range of this multifunctional iron oxide. In fact, these new findings may be an indication that the presumed multiferrroic properties of $\varepsilon-\mathrm{Fe}_{2} \mathrm{O}_{3}$ at room temperature actually could persist and extend also into the new ferrimagnetic FM1 phase.

\section{ACKNOWLEDGMENTS}

We thank financial support from the Spanish Ministry of Economy and Competitiveness, through projects MAT2015-686760-02-2-P, MAT2012-38213-C02-02, MAT2016-77391-R and "Severo Ochoa" Programme for Centres of Excellence in R\&D (SEV- 2015-0496 and SEV-2013-0295). The formers are co-funded by ERDF of European Union. The Generalitat de Catalunya is also acknowledged for financial support (projects 2014SGR213 and 2014SGR1015). ICN2 is funded by the CERCA Programme/Generalitat de Catalunya. We also acknowledge ILL and ALBA for granting beamtime. C. Ritter is acknowledged for technical assistance during neutron measurements. J.L.G-M thanks J. M. Pérez-Mato for fruitful discussions. Ana Arauzo, from the Physical Measurements service at University of Zaragoza is acknowledged for performing ac susceptibility measurements. 


\section{REFERENCES}

1. Cornell, R. M.; Schwertmann, U., Applications. In The Iron Oxides, Wiley-VCH Verlag GmbH \& Co. KGaA: 2004; pp 509-524.

2. Carraro, G.; Maccato, C.; Gasparotto, A.; Montini, T.; Turner, S.; Lebedev, O. I.; Gombac, V.; Adami, G.; Van Tendeloo, G.; Barreca, D.; Fornasiero, P., Enhanced Hydrogen Production by Photoreforming of Renewable Oxygenates Through Nanostructured $\mathrm{Fe}_{2} \mathrm{O}_{3}$ Polymorphs. Adv. Funct. Mater. 2014, 24, 372-378.

3. Tucek, J.; Zboril, R.; Namai, A.; Ohkoshi, S., $\varepsilon-\mathrm{Fe}_{2} \mathrm{O}_{3}$ : An Advanced Nanomaterial Exhibiting Giant Coercive Field, Millimeter-Wave Ferromagnetic Resonance, and Magnetoelectric Coupling. Chem. Mater. 2010, 22, 6483-6505.

4. $\quad$ Gich, M.; Fina, I.; Morelli, A.; Sánchez, F.; Alexe, M.; Gàzquez, J.; Fontcuberta, J.; Roig, A., Multiferroic Iron Oxide Thin Films at Room Temperature. Adv. Mater. 2014, 26, 4645-4652.

5. Zboril, R.; Mashlan, M.; Petridis, D., Iron(III) oxides from thermal processessynthesis, structural and magnetic properties, Mossbauer spectroscopy characterization, and applications. Chem. Mater. 2002, 14, 969-982.

6. Gich, M.; Roig, A.; Taboada, E.; Molins, E.; Bonafos, C.; Snoeck, E., Stabilization of metastable phases in spatially restricted fields: the case of the $\mathrm{Fe}_{2} \mathrm{O}_{3}$ polymorphs. Faraday Discuss. 2007, 136, 345-354.

7. Sakurai, S.; Namai, A.; Hashimoto, K.; Ohkoshi, S., First Observation of Phase Transformation of All Four $\mathrm{Fe}_{2} \mathrm{O}_{3}$ Phases $(\gamma \rightarrow \varepsilon->\beta \rightarrow \alpha$-Phase). J. Am. Chem. Soc. 2009, 131, 18299-18303.

8. Tadic, M.; Milosevic, I.; Kralj, S.; Mitric, M.; Makovec, D.; Saboungi, M. L.; Motte, L., Synthesis of metastable hard-magnetic $\varepsilon-\mathrm{Fe}_{2} \mathrm{O}_{3}$ nanoparticles from silica-coated akaganeite nanorods. Nanoscale 2017, 9, 10579-10584.

9. Tronc, E.; Chaneac, C.; Jolivet, J. P., Structural and magnetic characterization of $\varepsilon-$ $\mathrm{Fe}_{2} \mathrm{O}_{3}$. J. Solid State Chem. 1998, 139, 93-104.

10. Jin, B.; Ohkoshi, S.; Hashimoto, K., Giant coercive field of nanometer-sized iron oxide. Adv. Mater. 2004, 16, 48-51.

11. Popovici, M.; Gich, M.; Niznansky, D.; Roig, A.; Savii, C.; Casas, L.; Molins, E.; Zaveta, K.; Enache, C.; Sort, J.; de Brion, S.; Chouteau, G.; Nogues, J., Optimized synthesis of the elusive $\varepsilon-\mathrm{Fe}_{2} \mathrm{O}_{3}$ phase via sol-gel chemistry. Chem. Mater. 2004, 16, 5542-5548. 12. Namai, A.; Sakurai, S.; Nakajima, M.; Suemoto, T.; Matsumoto, K.; Goto, M.; Sasaki, S.; Ohkoshi, S., Synthesis of an Electromagnetic Wave Absorber for High-Speed Wireless Communication. J. Am. Chem. Soc. 2009, 131, 1170-1173.

13. Namai, A.; Yoshikiyo, M.; Yamada, K.; Sakurai, S.; Goto, T.; Yoshida, T.; Miyazaki, T.; Nakajima, M.; Suemoto, T.; Tokoro, H.; Ohkoshi, S., Hard magnetic ferrite with a gigantic coercivity and high frequency millimetre wave rotation. Nat. Commun. 2012, 3 , 1035 .

14. Lopez-Ortega, A.; Estrader, M.; Salazar-Alvarez, G.; Roca, A. G.; Nogues, J., Applications of exchange coupled bi-magnetic hard/soft and soft/hard magnetic core/shell nanoparticles. Phys. Rep. 2015, 553, 1-32.

15. Hozumi, T.; Irie, S.; Chiba, T. Magnetic Material, Magnet and Method for Producing the Magnetic Material, WO2012/101752 A1. 02.08.2012, 2012.

16. Gich, M.; Frontera, C.; Roig, A.; Fontcuberta, J.; Molins, E.; Bellido, N.; Simon, C.; Fleta, C., Magnetoelectric coupling in $\varepsilon-\mathrm{Fe}_{2} \mathrm{O}_{3}$ nanoparticles. Nanotechnology 2006, 17, $687-$ 691. 
17. Gich, M.; Roig, A.; Frontera, C.; Molins, E.; Sort, J.; Popovici, M.; Chouteau, G.; Marero, D. M. Y.; Nogues, J., Large coercivity and low-temperature magnetic reorientation in $\varepsilon-\mathrm{Fe}_{2} \mathrm{O}_{3}$ nanoparticles. J. Appl. Phys. 2005, 98, 044307.

18. Gich, M.; Frontera, C.; Roig, A.; Taboada, E.; Molins, E.; Rechenberg, H. R.; Ardisson, J. D.; Macedo, W. A. A.; Ritter, C.; Hardy, V.; Sort, J.; Skumryev, V.; Nogues, J., High- and low-temperature crystal and magnetic structures of $\varepsilon-\mathrm{Fe}_{2} \mathrm{O}_{3}$ and their correlation to its magnetic properties. Chem. Mater. 2006, 18, 3889-3897.

19. Tseng, Y. C.; Souza-Neto, N. M.; Haskel, D.; Gich, M.; Frontera, C.; Roig, A.; van Veenendaal, M.; Nogues, J., Nonzero orbital moment in high coercivity $\varepsilon-\mathrm{Fe}_{2} \mathrm{O}_{3}$ and lowtemperature collapse of the magnetocrystalline anisotropy. Phys. Rev. B 2009, 79, 094404.

20. Forestier, H.; Guiot-Guillain, G., A new ferromagnetic vairety of iron sesquioxide. $C$. R. Acad. Sci. (Paris) 1934, 199, 720-723.

21. Schrader, R.; Buttner, G., A New phase of Iron (III)-oxide - $\varepsilon-\mathrm{Fe}_{2} \mathrm{O}_{3}$. Z. Anorg. Allg. Chem. 1963, 320, 220-234.

22. Ohkoshi, S.; Namai, A.; Sakurai, S., The Origin of Ferromagnetism in $\varepsilon-\mathrm{Fe}_{2} \mathrm{O}_{3}$ and $\varepsilon-$ $\mathrm{GaxFe}_{2-\mathrm{x}} \mathrm{O}_{3}$ Nanomagnets. J. Phys. Chem. C 2009, 113, 11235-11238.

23. López-Sánchez, J.; Serrano, A.; Del Campo, A.; Abuín, M.; Rodríguez de la Fuente, O.; Carmona, N., Sol-Gel Synthesis and Micro-Raman Characterization of $\varepsilon-\mathrm{Fe}_{2} \mathrm{O}_{3}$ Microand Nanoparticles. Chem. Mater. 2016, 28, 511-518.

24. Fauth, F.; Boer, R.; Gil-Ortiz, F.; Popescu, C.; Vallcorba, O.; Peral, I.; Fullà, D.; Benach, J.; Juanhuix, J., The crystallography stations at the Alba synchrotron. Eur. Phys. J. Plus 2015, 130, 160.

25. Rodriguez-Carvajal, J., Recent Advances in Magnetic-Structure Determination by Neutron Powder Diffraction. Physica B 1993, 192, 55-69.

26. Aroyo, M. I.; Perez-Mato, J. M.; Capillas, C.; Kroumova, E.; Ivantchev, S.; Madariaga, G.; Kirov, A.; Wondratschek, H., Bilbao crystallographic server: I. Databases and crystallographic computing programs. Z. Kristallogr. 2006, 221, 15-27.

27. Aroyo, M. I.; Kirov, A.; Capillas, C.; Perez-Mato, J. M.; Wondratschek, H., Bilbao crystallographic server. II. Representations of crystallographic point groups and space groups. Acta Crystallogr. A 2006, 62, 115-128.

28. Perez-Mato, J. M.; Gallego, S. V.; Tasci, E. S.; Elcoro, L.; de la Flor, G.; Aroyo, M. I., Symmetry-Based Computational Tools for Magnetic Crystallography. Annu. Rev. Mater. Res. 2015, 45, 217-248.

29. Campbell, B. J.; Stokes, H. T.; Tanner, D. E.; Hatch, D. M., ISODISPLACE: a webbased tool for exploring structural distortions. J. Appl. Crystallogr. 2006, 39, 607-614.

30. Kuriki, A.; Moritomo, Y.; Xu, S.; Ohoyama, K.; Kato, K.; Nakamura, A., Diffuse scattering due to geometrical frustration in $\mathrm{Mn}_{3} \mathrm{O}_{4}$. J. Phys. Soc. Jpn. 2003, 72, 458-459. 
Table I. Atomic coordinates of $\varepsilon-\mathrm{Fe}_{2} \mathrm{O}_{3}$ obtained from SXRPD at $300 \mathrm{~K}$ and $510 \mathrm{~K}$.

\begin{tabular}{|l|lll|lll|}
\hline & \multicolumn{3}{|c|}{$\mathrm{T}=300 \mathrm{~K}$} & \multicolumn{3}{c|}{$\mathrm{T}=510 \mathrm{~K}$} \\
\hline Atoms & \multicolumn{1}{|c|}{$\mathrm{x}$} & $\mathrm{y}$ & \multicolumn{1}{c|}{$\mathrm{z}$} & $\mathrm{x}$ & $\mathrm{y}$ & \multicolumn{1}{c|}{$\mathrm{z}$} \\
\hline $\mathrm{Fe} 1$ & $0.1947(5)$ & $0.1497(3)$ & $0.5806(2)$ & $0.1928(6)$ & $0.1499(4)$ & $0.5813(2)$ \\
$\mathrm{Fe} 2$ & $0.6828(3)$ & $0.0323(2)$ & $0.7921(3)$ & $0.6823(4)$ & $0.0314(2)$ & $0.7918(3)$ \\
$\mathrm{Fe} 3$ & $0.8083(3)$ & $0.1592(2)$ & $0.3062(2)$ & $0.8105(4)$ & $0.1583(2)$ & $0.3079(2)$ \\
$\mathrm{Fe} 4$ & $0.1816(5)$ & $0.1538(3)$ & 0.00000 & $0.1819(6)$ & $0.1542(4)$ & 0.00000 \\
O1 & $0.9754(13)$ & $0.3276(5)$ & $0.4340(5)$ & $0.9739(14)$ & $0.3281(6)$ & $0.4345(6)$ \\
O2 & $0.5112(13)$ & $0.4933(8)$ & $0.4187(9)$ & $0.5030(14)$ & $0.4907(9)$ & $0.4227(13)$ \\
O3 & $0.6537(15)$ & $1.0011(7)$ & $0.1895(5)$ & $0.6533(18)$ & $0.9990(8)$ & $0.1859(5)$ \\
O4 & $0.1624(14)$ & $0.1622(8)$ & $0.1956(4)$ & $0.1614(16)$ & $0.1608(8)$ & $0.1959(4)$ \\
O5 & $0.8449(15)$ & $0.1657(8)$ & $0.6680(5)$ & $0.8450(17)$ & $0.1669(10)$ & $0.6698(5)$ \\
O6 & $0.5286(13)$ & $0.1590(9)$ & $0.9375(6)$ & $0.5386(14)$ & $0.1654(10)$ & $0.9366(7)$ \\
\hline & $\mathrm{a}=5.0967(2)$ & $\mathrm{b}=8.7953(3)$ & $\mathrm{c}=9.4770(3)$ & $\mathrm{a}=5.1133(2)$ & $\mathrm{b}=8.8163(4)$ & $\mathrm{c}=9.4822(4)$ \\
\hline
\end{tabular}

Agreement factors for SXRPD pattern at $300 \mathrm{~K}(510 \mathrm{~K})$ : $\mathrm{R}_{\mathrm{B}}=1.35(1.31), \mathrm{R}_{\mathrm{f}}=1.04(1.01)$, $\mathrm{R}_{\mathrm{Mag}}=4.45(4.48), \chi^{2}=4.6(4.8)$ 
Table II. Magnetic groups and refined magnetic moments in the ferrimagnetic FM1 $\left(\mathrm{T}_{\mathrm{N} 1}\right)$ and FM2 ( $\left.\mathrm{T}_{\mathrm{N} 2}\right)$ phases. The $\mathrm{d}, \mathrm{r}$ and $\mathrm{t}$ labels of the four iron sites refer to distorted, regular and tetrahedral polyhedra, respectively). The goodness factors are given in Fig. 3.

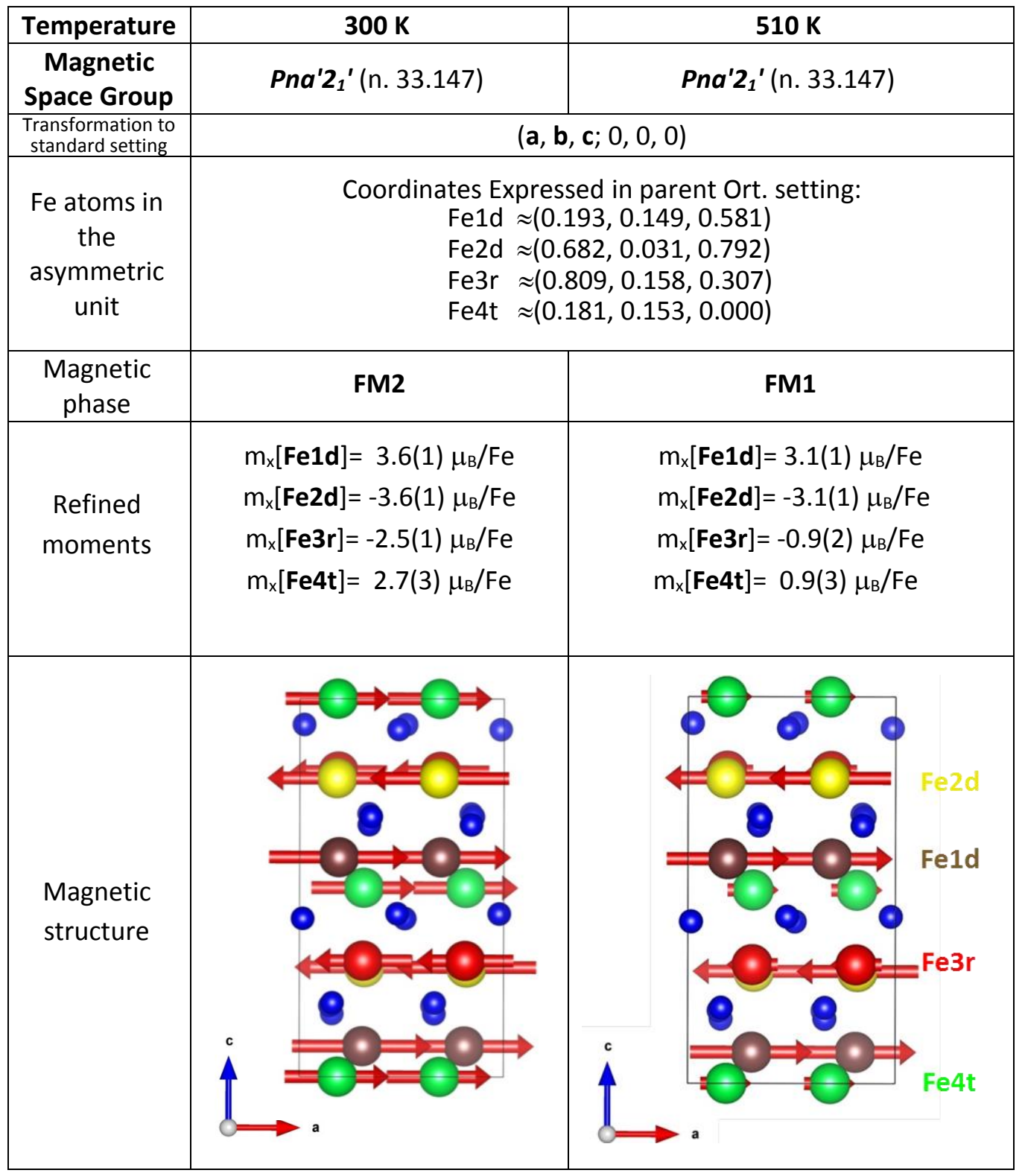


Table of Contents

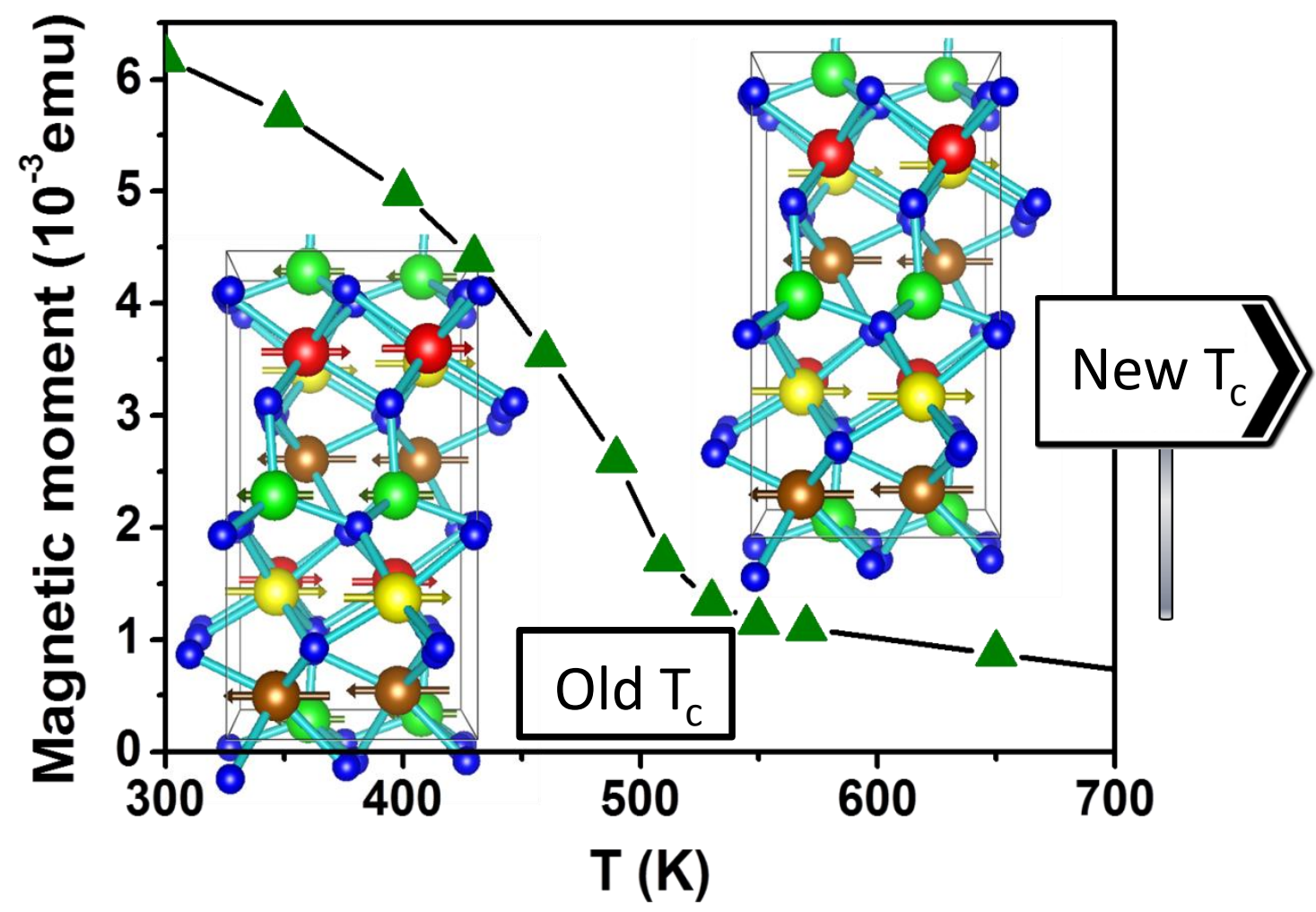

\section{ASSOCIATED CONTENT}

Supporting Information. Simulation of a neutron diffraction pattern of 50 wt. $\% \varepsilon-\mathrm{Fe}_{2} \mathrm{O}_{3}$ and 50 wt. $\% \alpha-\mathrm{Fe}_{2} \mathrm{O}_{3}$ compared to the experimental pattern collected at $305 \mathrm{~K}$. This material is available free of charge via the Internet at http://pubs.acs.org. 